\title{
Simulated low-intensity optical pulsar observation with single-photon detector (Research Note)
}

\author{
W. R. Leeb ${ }^{1}$, J. Alves ${ }^{2}$, S. Meingast ${ }^{2}$, and M. Brunner ${ }^{2}$ \\ ${ }^{1}$ Institute of Telecommunications, Vienna University of Technology, Gusshausstrasse 25, 1040 Wien, Austria \\ e-mail: walter.leeb@tuwien.ac.at \\ ${ }^{2}$ Department of Astrophysics, University of Vienna, Türkenschanzstrasse 17, 1180 Wien, Austria
}

Received 26 June 2014 / Accepted 27 November 2014

ABSTRACT

\begin{abstract}
Context. Optical radiation of pulsars offers valuable clues to the physics of neutron stars, which are our only probes of the most extreme states of matter in the present-day universe. Still, only about $1 \%$ of all cataloged pulsars have known optical counterparts. Aims. The goal of this work is to develop an observational method optimized for discovering faint optical pulsars.

Methods. A single-photon detector transforms the signal received by the telescope into a pulse sequence. The events obtained are time tagged and transformed into a histogram of event time differences. The histogram envelope presents the autocorrelation of the recorded optical signal and thus displays any periodicity of the input signal.

Results. Simulations show that faint pulsars radiating in the optical regime can be detected in a straightforward way. As an example, a fictitious pulsar with a $V$-magnitude of 24.6 mag and a signature like the Crab pulsar can be discovered within one minute using an 8-m class telescope. At the detector's peak sensitivity the average optical flux density would then amount to $F_{v}=0.63 \mu \mathrm{Jy}$. With a 40-m class telescope, such as the forthcoming European ELT, the detection of optical pulsars with magnitudes $V<30$ mag is within reach for a measurement time of one minute. A two-hour "blind search" with the ELT could reach $V \sim 31.3$ mag.

Conclusions. This method allows detecting faint periodic optical radiation with simple equipment and easy signal processing.
\end{abstract}

Key words. instrumentation: detectors - pulsars: general - pulsars: individual: Crab pulsar (PSR 0513+21) - methods: data analysis

\section{Introduction}

Pulsars are powerful tools for fundamental physics and astrophysics. Characterizing and understanding pulsars is key to such fundamental topics as extrasolar planet science, understanding the role of magnetic fields and turbulence in core-collapse physics, identifying the equation-of-state of superdense matter, detecting a cosmological gravitational wave background, and strong-field tests of gravity (e.g. Cordes et al. 2004). Multiwavelength observations are crucial for characterizing the neutron star spectral energy distribution and understanding the physics of the radiation processes in pulsar magnetospheres (e.g. Mignani 2012). Still, pulsars are intrinsically faint objects at optical wavelengths, and only about $1 \%$ of the approximately 2000 pulsars currently cataloged have known optical counterparts.

Recently we have developed a method of searching for faint periodic laser pulses as they might have been transmitted by extraterrestrial intelligence (ETI) and would eventually be received on Earth (Leeb et al. 2013). In essence, it consists of employing a single-photon detector followed by a time-tagging device. After recording the time-tagged events, one establishes a histogram of event-time differences. The histogram itself will show periodic peaks, from which the period of the incoming optical signal can be read, and the existence of extraterrestrial intelligence can be inferred. We now apply this method to a simulation of the observation of pulsars. The main difference is the period $T$ of the incoming optical signal: while we were assuming rates of
$\mathrm{kHz}$ to $\mathrm{MHz}$ for the ETI signals, we now deal with periods of typically $100 \mathrm{~Hz}$ or less.

In recent years a group from the University of Padova, Italy, had used single photon detectors in the visible for highly accurate timing analysis of the Crab pulsar's period and its phase stability (Naletto et al. 2009; Germanà et al. 2012; Zampieri et al. 2014). However, their method is based on flux time series obtained by counting the photon events in short, consecutive periods and putting them into time bins. The same principle is also applied with conventional measurements at radio frequencies, where the analog radio signal is digitized into time bins. In both cases this first step yields time series of the received optical power. A second step aims to detect any periodicity, e.g., by fast Fourier transform. Third, the so-called epoch-folding technique can be employed where the signal-to-noise ratio of the pulsar signal will be greatly improved and which may already include an estimation of the periodicity (Larsson 1996).

In the gamma-ray regime, the search for pulsars was quite successful using a "time-differencing technique" (Atwood et al. 2006; Ziegler et al. 2008). As is common, the first step is to bin the photon arrival times into time slots of width chosen with regard to the maximum expected pulsar frequency. In a second step, one takes those bins whose time difference is less than a chosen fraction (e.g., 1/16) of the measurement time. Lastly one applies a discrete Fourier transform on the differences between the photon numbers in all those bins in order to find any periodicity. 
The method presented below relies on the determination of event time differences as the very first step after time tagging each detected photonic event. In general, all ${ }^{1}$ of the calculated time differences are then grouped into time bins and can be displayed in a histogram. The histogram envelope represents the autocorrelation function of the entire light curve received during the measurement interval. It will thus contain a quasi-periodicity with a period equal to the pulsar light curve and a number of periods equal to the number of pulsar periods recorded. In a further step, the signal-to-noise ratio of the autocorrelation of a single pulsar period can be improved by folding the histogram periods, i.e., by a method equivalent to epoch folding. This procedure allows the entire information conveyed by the detected photons to be used conveniently (except, of course, the exact wavelength). It turns out that the folded autocorrelation thus obtained has a better signal-to-noise ratio than the folded light curve, so it may be preferred when the detection of a weak optical pulsar is the prime objective.

In Sect. 2 we describe the setup required for recording the time instants of photon-induced events and the generation of histograms that will disclose any periodicity of the signal received. As an example, Sect. 3 simulates the reception of the optical radiation from the Crab pulsar with a $0.8 \mathrm{~m}$ telescope employing standard equipment. In Sect. 4 we apply our method to fictitious pulsars of $V$-magnitude 24.6 and 30 mag using the $8.2 \mathrm{~m}$ VLT and a future $40 \mathrm{~m}$ telescope (ELT), respectively, and demonstrate the detectability of the periodic signal after a measurement time as short as $60 \mathrm{~s}$.

\section{Setup and data analysis}

Figure 1 sketches the setup we suggest employing to search for pulsars operating in the visible and near infrared $(0.4<\lambda<$ $1.0 \mu \mathrm{m})$. The pulsar radiation collected by a telescope is detected by a single-photon avalanche detector (SPAD), operated in the Geiger mode ${ }^{2}$. Because of the faintness of the optical signal, for each pulsar period the SPAD may produce only a few pulsarinduced output events manifested by electrical pulses. Next, the electrical output pulses are time-tagged at their rising edge, and each event is stored in a computer in the form of its relative arrival time, $t_{i}$. The total measurement time may range from about one hundred to several thousand pulsar periods $T$. (For the Crab pulsar detection presented as an example in Sect. 3 the number of periods was chosen to be $P=40$, asking for a measurement time of $1.35 \mathrm{~s}$ ). Lastly, data processing consists of calculating a histogram that shows the frequency $\mathrm{H}$ of all possible positive event-time differences $t_{i, j}=t_{i}-t_{j}$ in classes of properly chosen bin widths $b w$. The histogram envelope is the autocorrelation function of the optical input signal for positive time shifts.

In Fig. 2 we illustrate the formation of the histogram by means of a simple example: we assume the pulsar signal to be a single, smooth pulse $s(t)$ within an otherwise empty time period $T$, where we chose a ratio of pulse width to $T$ of

\footnotetext{
1 In case of insufficient computer capabilities, the time differences considered may be restricted to a value below the measurement time, as applied in Leeb et al. (2013). This approach would correspond to the method of Atwood et al. (2006), however, still with the basic difference that they manipulate photon numbers, while we manipulate times of photon arrivals. Alluding to the title of the paper by Atwood et al. (2006), our technique could be called the "truly time-difference technique".

2 Of course, any other detector type capable of single-photon detection with sufficiently short dead time, low dark count rate, and good quantum efficiency would do as well.
}

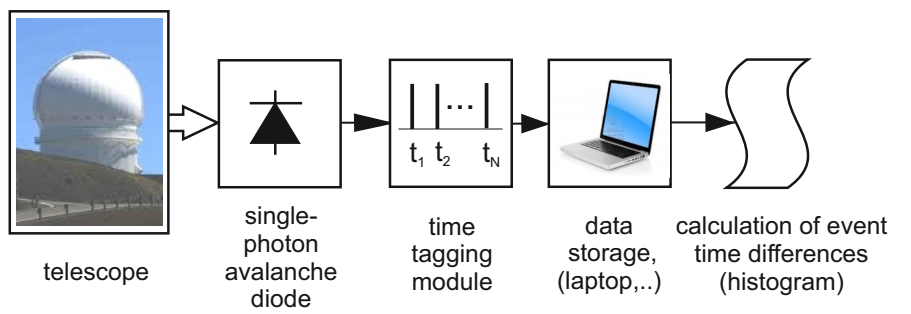

Fig. 1. Setup for detection and analysis of optical pulsar radiation.

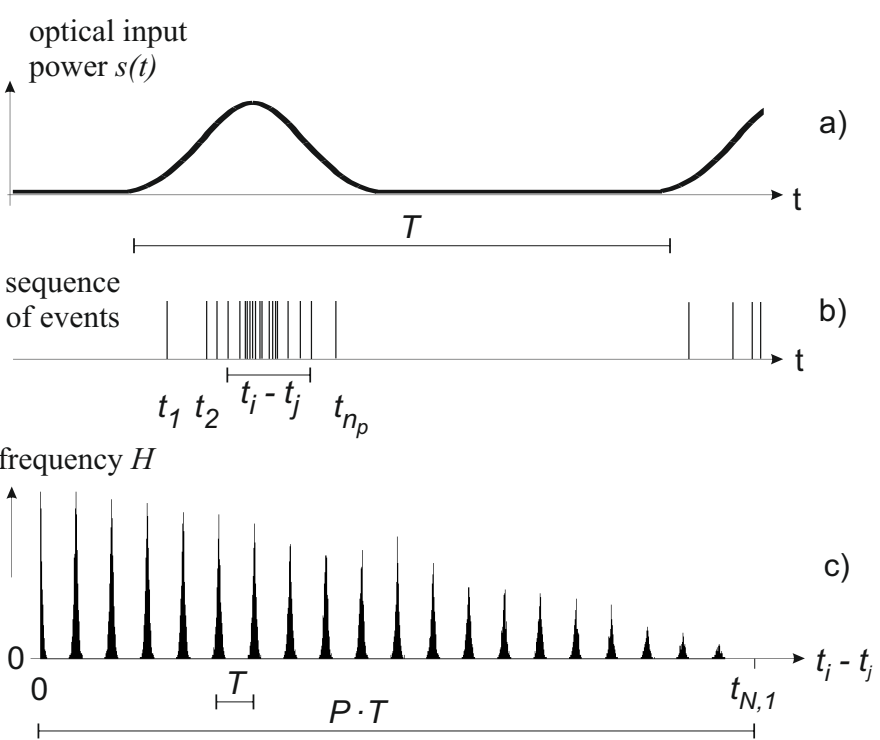

Fig. 2. a) Optical signal with period of length $T$ at the detector; b) one realization of pulsar-induced events; c) histogram obtained from the recording of $P$ periods. (Zero noise events assumed).

approximately 0.2 (see Fig. 2a). The combination of SPAD and time-tagging module may output a sequence of events as indicated in Fig. 2b. Specifically, we assumed $n_{\mathrm{p}}=20$ events per period $T$; i.e., the optical input consists of $20 / \eta$ photons per period, where $\eta$ is the detector quantum efficiency. We further assumed that we had recorded the pulsar signal for $P=20$ periods. In each of these periods, the events will occur in a statistically varying manner. However, averaged over many periods, the temporal density of events is proportional to the signal strength $s(t)$. For generating the histogram, we calculated all possible combinations of $t_{i, j}$ with

$j=1,2, \ldots, N-1$ and $i=j+1, j+2, \ldots, N$

where $N=n_{\mathrm{p}} \cdot P$ is the index of the last event. The number of time differences is given by

$Y_{N}=\frac{N^{2}-N}{2}$

and amounts to $Y_{N}=79800$ in the present example. Figure 2c shows the resulting histogram in case one chooses the number of bins to be $B=t_{N, 1} / b w=20000$. The periodicity $T$ of the signal $s(t)$ manifests itself in the regular spacing of the histogram peaks. Because of the statistical nature of the events, their peaks's height does not decrease strictly linearly. However, their mutual time distance equals $T$. Of course, pulsar events will be accompanied by noise events caused by background photons and detector imperfections. In the histogram this will add a noise floor with an envelope decreasing linearly along the horizontal axis. 


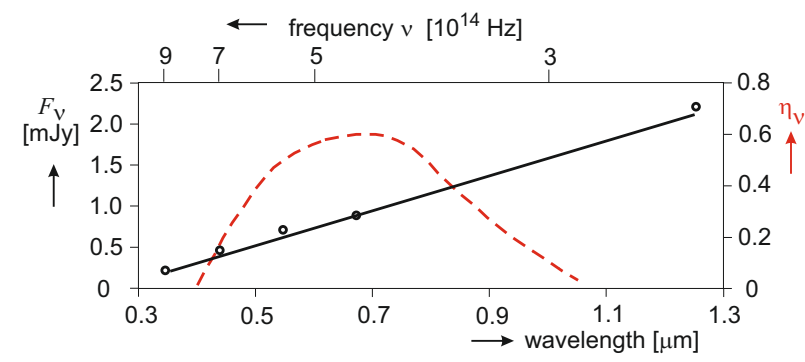

Fig. 3. Observed flux density $F_{v}$ of the Crab pulsar (left ordinate). The circles are the measured values reported by Eikenberry et al. (1997), and the full line the (linear) approximation used in this paper. The dashed line gives the typical quantum efficiency $\eta_{v}$ of detectors (right ordinate).

\section{Simulation of Crab pulsar detection with a $0.8 \mathrm{~m}$ telescope}

\subsection{Simulation of expected single-photon events}

In this section we present our simulation of the sequence of events expected at the output of the time-tagging module (see Fig. 1) when a telescope with diameter $D_{R}=0.8 \mathrm{~m}$ is directed toward the pulsar in the Crab nebula (magnitude $V=16.6 \mathrm{mag}$ ). For the single-photon detector we take typical specifications of single-photon counting modules that are sensitive between 400 and $1050 \mathrm{~nm}^{3}$. The time-tagging module is assumed to be of the type developed by $\mathrm{AIT}^{4}$, which for the present application, provides abundant time resolution and by far sufficiently low dead time.

As a first step we estimate the number of events, $n$, to be generated in one pulsar period. Figure 3 shows five values of the observed time-averaged flux density $F_{v}$ (Percival et al. 1993; Eikenberry et al. 1997). The figure also includes a linear approximation to the measured values, since we use it for the numerical calculation. Furthermore, we have included the typical wavelength dependence of the quantum efficiency, $\eta_{\nu}$, of the single-photon counting detectors that we have in mind. The number of pulsar-induced events may be calculated easily using

$n_{\mathrm{p}}=t r_{\mathrm{pu}} \cdot \frac{D_{R}^{2} \pi T}{4 h} \int_{v_{l}}^{v_{u}} \frac{F_{v} \eta_{v}}{v} \mathrm{~d} v$

where $h$ is Planck's constant and $v$ the light frequency (or indicates a frequency dependence). Specifically, $v_{1}$ and $v_{\mathrm{u}}$ are the lower and upper frequency limits of the detector, corresponding to $\lambda_{\mathrm{u}}=1050 \mathrm{~nm}$ and $\lambda_{1}=400 \mathrm{~nm}$, respectively, in our specific case. The factor $t r_{\mathrm{pu}}$ accounts for the pulsar signal transmission of the atmosphere and losses in the receiving instrument. It also covers a luminosity decrease of $6 \%$, following from a reduction of $3 \mathrm{mmag} / \mathrm{year}{ }^{5}$ since 1991 (Sandberg \& Sollerman 2009), when the fluxes were measured. Very conservatively, we put the total value ${ }^{6}$ at $t r_{\mathrm{pu}}=0.45$. For the parameter values cited and for

\footnotetext{
3 See, e.g., http://www.micro-photon-devices.com/Products or http://www . perkinelmer.com/PDFS/downloads/dts_ spcm-aq4c.pdf

4 Austrian Institute of Technology, data sheet at http://www.ait.ac.at/research-services/ research-services-safety-security/ optical-quantum-technologies/

5 This value is attached with a relatively large uncertainty of $\pm 55 \%$.

6 This low value is due to the assumption of atmospheric losses as high as $t r_{\text {atm }}=0.5$ for an observatory at low sea level and an instrument transmission of $t r_{\text {inst }}=0.9$.
}

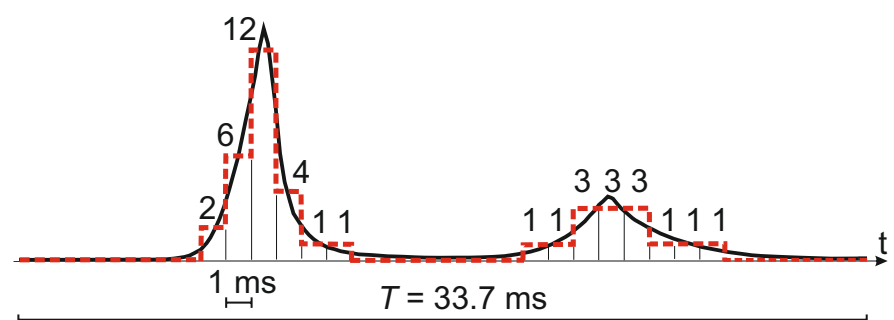

Fig. 4. Sketch of Crab pulsar intensity in the visible and near-infrared, (Percival et al. 1993, full line) and approximating digitization (dashed line). The numbers on top of each step give the number of events within $1 \mathrm{~ms}$ when partitioning a total of 40 events.

the present period of $T=33.7 \mathrm{~ms}^{7}$, Eq. (3) yields $n_{\mathrm{p}}=40$ events per pulsar period. The optical light curve of the Crab pulsar is presented well by the two-peak characteristic given in Fig. 4, not only in the visible range (400 to $700 \mathrm{~nm}$ ) but also in $J$-band $(1100 \mathrm{~nm}$ to $1390 \mathrm{~nm})$. When simulating the time instants at which the events will occur within each pulsar period, we have to recall that a) the density of events should mirror the intensity of the pulsar's light curve; and b) these time instants should occur randomly. To accommodate the 40 events, we approximate the two peaks by the two step functions.

The step widths were chosen to be $1 \mathrm{~ms}$, and the height of each step is proportional to the number of events to occur within that particular step at random instants of time. In the figure, the number on top of each step gives the number of events within that millisecond. As a result, the first peak will consist of 26 events and the second peak of 14 events $^{8}$. For the exemplary numerical simulation considered in this section, we generate $P=40$ such periods mutually separated by the duration $T$, each consisting of $n_{\mathrm{p}}=40$ time instants. Finally, noise events are simulated by adding events randomly distributed within the length $P \cdot T$ of the entire sequence. The amount of noise may be conveniently introduced via a rate of noise events, $n_{\mathrm{n}}$, expressed in number per second. One may distinguish between background-induced noise rates caused by sky background $\left(n_{\mathrm{SB}}\right)$ and by nebular background $\left(n_{\mathrm{NB}}\right)$, and a dark count rate of the single-photon detector, $n_{\mathrm{D}}$. The total number of events is thus

$N=n_{\mathrm{p}} \cdot P+P \cdot T \cdot n_{\mathrm{n}}$

where the first term to the right gives the number of pulsar events and the second term that of the noise events, with

$n_{\mathrm{n}}=n_{\mathrm{SB}}+n_{\mathrm{NB}}+n_{\mathrm{D}}$

We now assume an observatory location yielding a (rather unfavorable) night sky magnitude of $R=17 \mathrm{mag} / \mathrm{arcsec}^{2}$ and consider that the crab nebula has a brightness of $2.9 \times$ $10^{-16} \mathrm{erg} \mathrm{cm}^{-2} \mathrm{~s}^{-1} \AA^{-1} \operatorname{arcsec}^{-2}$ in the $V$-band and a spectral index of 0.6 , both in the immediate neighborhood of the pulsar (Véron-Cetty \& Woltjer 1993). For a field of view of the SPAD 9 of fov $=1$ arcsec and a filter with a bandwidth $\Delta \lambda=650 \mathrm{~nm}$ centered at $\lambda=0.7 \mu \mathrm{m}$, one obtains $n_{\mathrm{SB}}=2270 \mathrm{~s}^{-1}$ and

7 Jodrell Bank Crab Pulsar Monthly Ephemeris, available online at www.jb.man.ac.uk/pulsar/crab/html

8 This ratio of energy between the two peaks of 26/14 $=1.86$ agrees nicely with the one given in Eikenberry et al. (1997), if one assumes a misprint in their Fig. 9 (peak2/peak1 instead vice versa).

9 Here we assume that proper measures have been taken to reduce the field of view of the detector to the lowest possible value compatible with the telescope resolution, but high enough to collect most of the light radiated by a distant point source, i.e. the pulsar. 


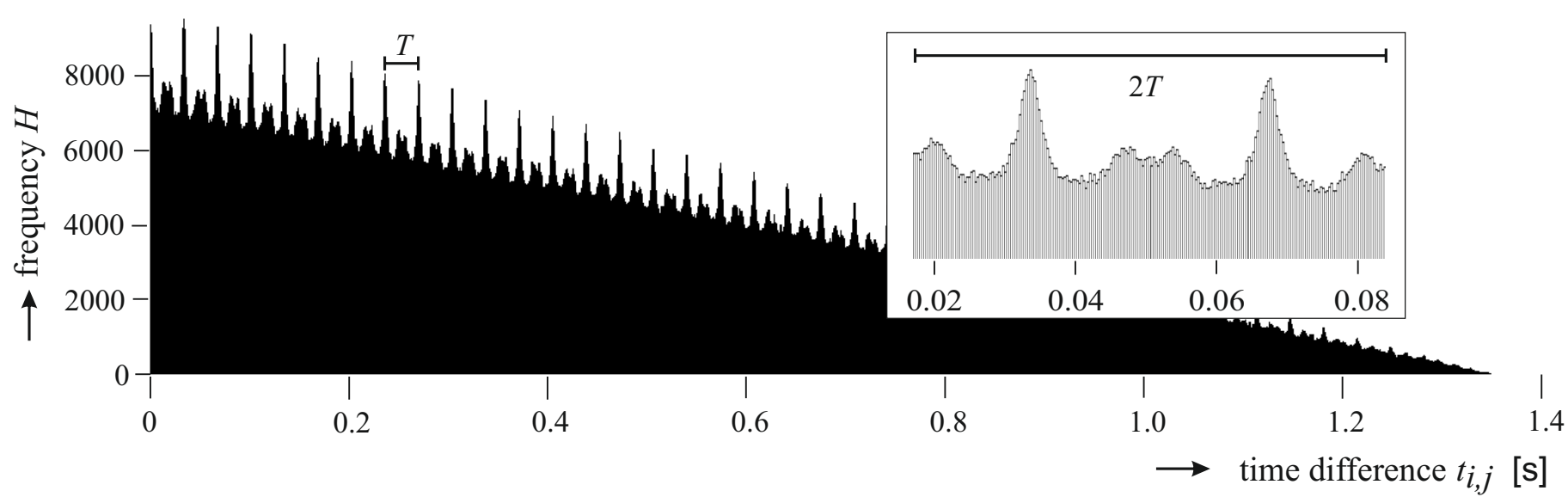

Fig. 5. Histogram obtained for simulated detection of the Crab pulsar ( $V$ magnitude 16.6). The inset shows a stretched upper detail of a section from the left of the diagram (telescope diameter $D_{R}=0.8 \mathrm{~m}$, measurement time $=1.35 \mathrm{~s}$, rate of noise events $n_{\mathrm{n}}=3120 \mathrm{~s}^{-1}$, number of pulsar events per period $n_{\mathrm{p}}=40$, pulsar signal transmission $t r_{\mathrm{pu}}=0.45$, bin width $b w=0.3 \mathrm{~ms}$ ).

$n_{\mathrm{NB}}=800 \mathrm{~s}^{-1} 10$. With a dark count rate of $n_{\mathrm{D}}=50 \mathrm{~s}^{-1}$ for single-photon detectors currently available we arrive at a total noise rate of $n_{\mathrm{n}}=3120 \mathrm{~s}^{-1}$. For the total number of events, Eq. (4) yields $N=5806$.

\subsection{Resulting histogram}

With the specific time sequence established above we now generate a histogram of all possible positive event time differences $t_{i, j}$, as described in Sect. 2 . The resulting histogram for one realization, as obtained by using the statistics software " $R$ "11, is shown in Fig. 5, where we chose a bin width of $b w=3 \times 10^{-4} \mathrm{~s}$, yielding $B \approx 4500$ bins. The mutual separation of the main peaks can easily be determined to be $0.0337 \mathrm{~s}$, identical to the pulsar period $T$. The measurement time in the example given was only $1.35 \mathrm{~s}$. While the dominating peaks are a result of the autocorrelation of the first peak in the pulsar's light curve with itself, the double peaks in between stem from the autocorrelation of the first peak with the second peak (compare Fig. 4). The (triangular) histogram noise floor is caused by the noise events. In Appendix A we present a quantitative characterization of the signal-to-noise ratio in the histogram bin at $t_{i, j}=T$, i.e., the first of the bins indicating the existence of a periodicity of the optical input signal $s(t)$.

As soon as the length of the pulsar period can be estimated, a further step toward obtaining a more accurate shape of the autocorrelated input signal $s(t)$ can be done by capitalizing on the $P$-fold information contained in the histogram envelope. To this end the values of frequency $H$ are superimposed, block by block of length $T$. The improvement of the signal-to-noise by superimposing $k$ periods $(1 \leq k \leq P)$ is quantified in Appendix B and demonstrated in Fig. 6a for the example discussed (here $k=P=40)^{12}$. For comparison, we also computed the folded light curve that would be obtained by conventional photon counting, i.e. based on flux time series, as mentioned in the introduction (see Fig. 6b). Of course, the diagrams of Fig. 6 present different functions. But when searching for a periodicity, the one

\footnotetext{
${ }^{10}$ Here we took into account that the sky background is attenuated by the instrument transmission $t r_{\text {inst }}=0.9$ and the nebula background by the product of atmospheric transmission $t r_{\text {atm }}=0.5$ and $t r_{\text {inst }}$.

${ }^{11} \mathrm{R}$ programming language, available online at $\mathrm{http}: / / \mathrm{wWw}$. r-project.org

12 The required accuracy of the knowledge of $T$ is on the order of $b w / k$.
}

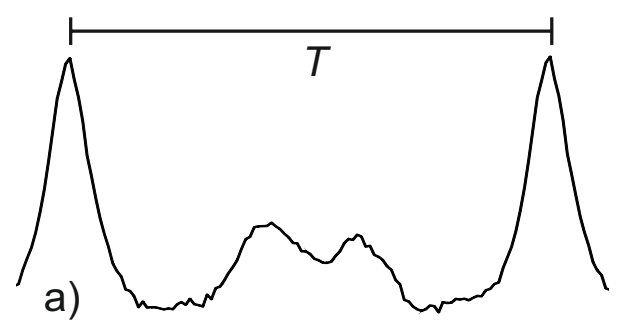

bins of TOA differences (folded)

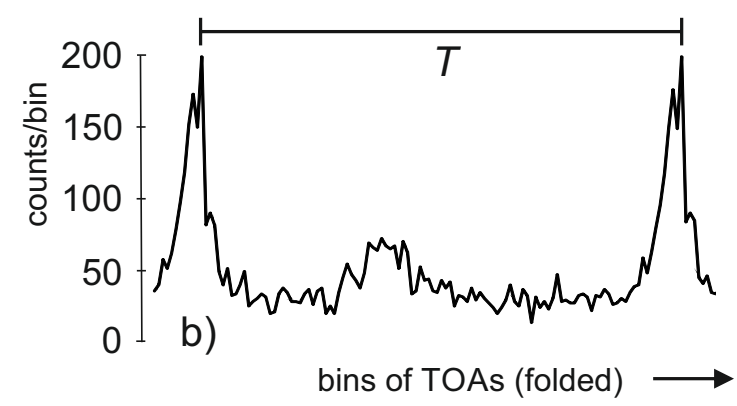

Fig. 6. a) Autocorrelation of the optical pulsar flux obtained by piecewise superimposing the envelope of the histogram of Fig. 5; b) detected optical flux versus time, as obtained by folding all the periods of the observing run and taking the average. The detected photon time series were identical for a) and b), and the pulsar period $T$ was divided into 112 bins in both cases. Clearly, method a) provided the better signal-to-noise ratio. (TOA ... time of arrival of photons).

based on the histogram of time differences offers an increased signal-to-noise ratio, hence better sensitivity.

\section{Extension to fainter pulsar using larger telescopes}

When searching for pulsars of larger magnitude, one may arrive at a sufficiently high histogram signal-to-noise ratio by using a larger telescope, by reducing the spectrum investigated and the field-of-view, or by increasing the number of pulsar periods recorded by lengthening the measurement time, as demonstrated in the following sections. 


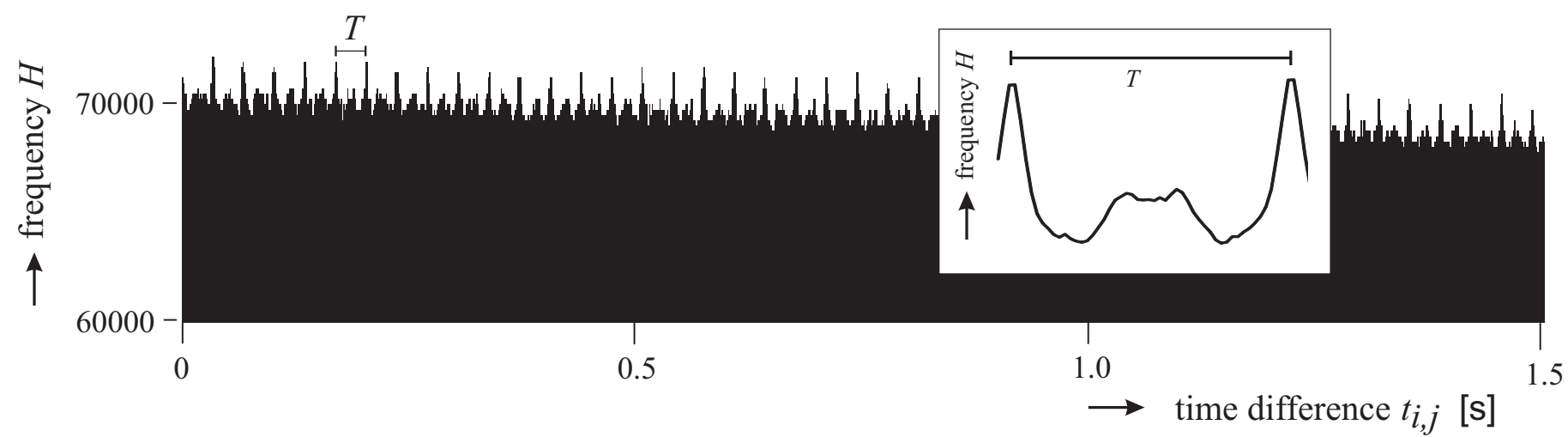

Fig. 7. Part of the histogram (with the noise floor mostly cut off) obtained from simulated detection of a Crab-like pulsar but with a flux eight magnitudes fainter; i.e., $V$-magnitude $=24.6$. (telescope diameter $D_{R}=8.2 \mathrm{~m}$, measurement time $\approx 1 \mathrm{~min}$, field-of-view fov $=0.6$ arcsec, rate of noise events $n_{\mathrm{n}}=1210 \mathrm{~s}^{-1}$, number of pulsar events per period $n_{\mathrm{p}}=3.55$, pulsar signal transmission $t r_{\mathrm{pu}}=0.8$, bin width $b \mathrm{w}=0.674 \mathrm{~ms}$ ). The inset shows the envelope for one period after superimposing the first $k=241$ consecutive blocks of length $T$ of the histogram.

\subsection{Very Large Telescope (VLT)}

As a telescope we suppose to use one of ESO's Very Large Telescopes (VLT) with a diameter of $D_{R}=8.2 \mathrm{~m}$. Because of its location we may assume a pulsar signal transmission as high as $t r_{\mathrm{pu}}=0.8$. We simulated the pulsar events received from a fictitious pulsar with a light curve and spectral distribution identical to that of the Crab pulsar, but of $V$-magnitude 24.6, so eight magnitudes fainter. At a wavelength of $\lambda=0.7 \mu \mathrm{m}$, the flux density now amounts to as little as $F_{v}=0.63 \mu \mathrm{Jy}$. This time we apply a filter bandwidth $\Delta \lambda=350 \mathrm{~nm}$, again centered at $\lambda=0.7 \mu \mathrm{m}$, and assume that the image quality is such that the field of view can be reduced to 0.6 arcsec. Instead of the $n_{\mathrm{p}}=40$ events per period expected for the real Crab pulsar (see Sect. 3.1) the average number of pulsar events per period will be as low as $n_{\mathrm{p}}^{\prime}=3.55$, as calculated using Eq. (3). For simulating a sequence of pulsar event time instants, we may still use the scheme shown in Fig. 4 for $n_{\mathrm{p}}=40$ by randomly picking out events with a probability of $p=n_{\mathrm{p}}^{\prime} / n_{\mathrm{p}}=0.089$.

The observatory location on Paranal can yield a night sky magnitude of $R=21 \mathrm{mag} / \operatorname{arcsec}^{2}$ (Patat 2003), which corresponds to a rate of some $n_{\mathrm{SB}}=1160 \mathrm{~s}^{-1}$ for the sky-background events in case of fov $=0.6$ arcsec and an instrument transmission of $t r_{\text {inst }}=0.9$. For any pulsar that is not as extremely young as the Crab pulsar, the originally accompanying nebula will most likely have already dispersed. Thus we only have to add the dark rate of $n_{\mathrm{D}}=50 \mathrm{~s}^{-1}$ and arrive at a total noise rate of $n_{\mathrm{n}}=1210 \mathrm{~s}^{-1}$.

To improve the signal-to-noise ratio, we then record $P=$ 1800 periods. The resulting histogram extends up to $t_{i, j}=$ $1800 T=60.7 \mathrm{~s}$, and the upper part of the first $1.5 \mathrm{~s}$ is displayed in Fig. 7. The existence of a second pulsar peak (cf. Fig. 4) can only be suspected. The histogram bin width was chosen to be $b w=0.674 \mathrm{~ms}$. In this scenario the (typical) number of pulsar events within the measurement time was 6400, that of noise events was 73400 . As above, one can improve on the histogram envelope by superimposing $k$ successive blocks of length $T$. The result shown in the inset of Fig. 7 (with $k=241)^{13}$ not only reveals the first pulsar peak but also the existence of the second peak. Owing to the choice of $b w=T / 50$, the graph in the inset

\footnotetext{
13 To obtain the inset, only $k=241$ blocks were superimposed, while the entire histogram consists of $P=1800$ blocks. This already yields a signal-to-noise ratio improvement of $50 \%$ of that maximally achievable when choosing $k=P$ (see Appendix B).
}

is composed of 50 discrete values; i.e., the autocorrelated input signal has a temporal resolution of $T / 50$.

\section{2. $40-m$ class telescope}

Here we consider the use of a 40-m class telescope, such as is presently under discussion - and possibly available in a decade from now - in the form of the Extremely Large Telescope (ELT). We again assume a Crab-pulsar-like input signal, but now aim at a source of magnitude $V=30 \mathrm{mag}$, corresponding to a flux density of $F_{v}=11 \mathrm{nJy}$ at $\lambda=0.7 \mu \mathrm{m}$. For a transmission of $t r_{\mathrm{pu}}=0.8$, one will observe $n_{\mathrm{p}}^{\prime}=0.585$ pulsar events per period $T$ when choosing a filter bandwidth of $\Delta \lambda=350 \mathrm{~nm}$. As before, the night sky magnitude of $R=21 \mathrm{mag} / \mathrm{arcsec}^{2}$ will determine the noise events. We now assume that the entire point spread function of the pulsar fits into a field of view as small as fov $=0.1$ arcsec. Then the noise rate becomes $n_{\mathrm{n}}=820 \mathrm{~s}^{-1}$.

For a measurement time of one minute (i.e., 1800 pulsar periods), visual inspection of the histogram established with a bin width of $b w=1.685 \mathrm{~ms}$ does not reveal any periodicity, as shown in Fig. 8, which presents the histogram's first $0.5 \mathrm{~s}$ of time differences. However, when superimposing the first $k=700$ blocks of length $T^{14}$, the resulting envelope even indicates the existence of a smaller, second pulsar peak, as demonstrated by the inset. Here, because of the choice of $b w=T / 20$, the graph in the inset has a temporal resolution of $T / 20$.

Equation (A.2) of Appendix A allows drawing a plot that gives the required pulsar events per period, $n_{\mathrm{p}}$, to achieve a prescribed signal-to-noise ratio $S N_{\text {Hist }}$ in the histogram as a function of measurement time $M=P \cdot T$, as exemplified in Fig. 9. We again assumed a Crab-like pulsar $(T=33.7 \mathrm{~ms})$ and a histogram bin width $b w=T / 20$, and assumed an effective width of the first pulsar peak of $d=3 \mathrm{~ms}$. The symbol $y$ in the quantity given by the ordinate stands for the fraction of $n_{\mathrm{p}}$ leading to the main autocorrelation peaks; i.e., $y=0.65$ for the Crab pulsar. The plot is drawn for four values of background rates $n_{\mathrm{n}}$, which could represent the different fields of view required for different or varying point spread functions. To a very good approximation, one observes a dependence $n_{\mathrm{p}} \cdot y / \sqrt{S N_{\text {Hist }}} \propto M^{-0.25}$. The black circle corresponds to the example presented in Fig. 8 $\left(n_{\mathrm{p}}=0.585, S N_{\text {Hist }}=0.55\right)$. As indicated by the vertical arrow, for a pulsar $1.3 \mathrm{mag}$ fainter (corresponding to $V=31.3 \mathrm{mag}$

\footnotetext{
${ }^{14}$ Such superposition requires the a priori knowledge of period $T$. For a histogram as in Fig. 8, $T$ can be found by a combination of Fourier and correlation analysis.
} 


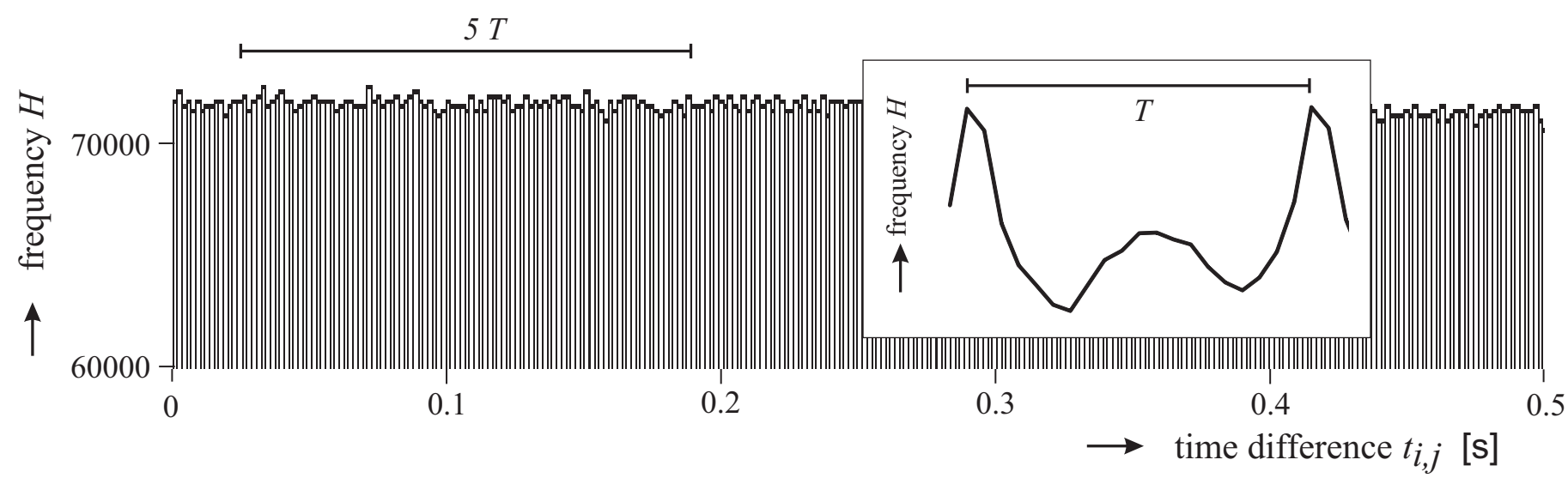

Fig. 8. As in Fig. 7, but for telescope diameter $D_{R}=40 \mathrm{~m}$, pulsar magnitude $V=30 \mathrm{mag}$, number of pulsar events per period $n_{\mathrm{p}}^{\prime}=0.585$, field of view $f o v=0.1 \mathrm{arcsec}$, rate of noise events $n_{\mathrm{n}}=820 \mathrm{~s}^{-1}$, bin width $b w=1.685 \mathrm{~ms}$. The inset presents the envelope after superimposing the first 700 consecutive blocks of length $T$.

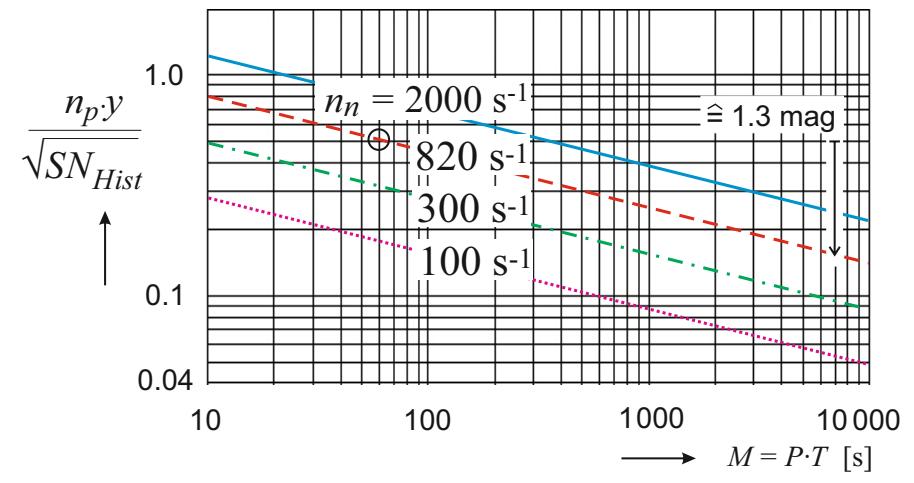

Fig. 9. Normalized number of required pulsar events per pulsar period, $n_{\mathrm{p}}$, to achieve a prescribed signal-to-noise ratio in the histogram, $S N_{\text {Hist }}$, as a function of measurement time $M=P \cdot T$ for four values of background photon rates $n_{\mathrm{n}}$. Period $T=33.7 \mathrm{~ms}$ and bin width $b w=T / 20$ are the same as for Fig. 8. The effective width of the first pulsar peak is taken as $d=3 \mathrm{~ms}$ (compare Appendix A). The black circle indicates the case of Fig. 8; i.e., $n_{\mathrm{p}}=0.585, S N_{\text {Hist }}=0.55$, fraction of $n_{\mathrm{p}}$ leading to histogram peak: $y=0.65$.

and $\left.n_{\mathrm{p}}=0.154\right)$, the measurement time $M$ would have to be increased to two hours ${ }^{15}$ to achieve the same $S N_{\text {Hist }}$. However, for the autocorrelation obtained after superimposing all available histogram blocks of length $T$, the then relevant quantity $n_{\mathrm{p}} \cdot y / \sqrt{S N_{\text {Sup }, P}}$ (compare Eq. (B.1)) has a $M^{-0.5}$ dependence! For the cases considered in this paper, the background rate $n_{\mathrm{n}}$ depends heavily on the field of view, fov. If the sky background dominates the total noise count rate, $n_{\mathrm{n}} \propto f o v^{2}$, then the influence of a change of fov is described in Fig. 9 by properly adapting $n_{\mathrm{n}}$.

\section{Summary and outlook}

We described a method of observing pulsars emitting in spectral regimes where single-photon detectors are available. A singlephoton avalanche diode followed by a time-tagging device constitutes the simple - though sensitive - equipment. The data processing employed corresponds to forming the autocorrelation of the received light curve. The resulting histogram of the time differences of single-photon events reveals the existence (and the value) of any periodicity.

\footnotetext{
${ }^{15}$ Clearly, signal processing would then require drastically increased calculation time or computer capacity.
}

As an example, we demonstrated that the Crab pulsar can easily be detected within one second with a $0.8 \mathrm{~m}$ telescope. We also showed that with a VLT-class telescope and a measurement time of one minute, the Crab pulsar could be detected if it had an eight times fainter magnitude. The anticipated realization of a $40 \mathrm{~m}$ telescope in the form of the ELT will allow a search for optical pulsars of magnitude fainter than $V=30 \mathrm{mag}$, especially if a resolution down to 0.1 arcsec can be obtained. When observing from a ground-based observatory, the limiting factor for the proposed method is the brightness of the night sky. A space telescope could very well be an advantage, even if it is much smaller than an Earth-bound instrument. In any case, the telescope does not have to be diffraction limited, because its task is only to concentrate the radiation collected onto the detector's sensitive area. But, of course, a poor optical quality will result in increased background noise. Using fiber-optic devices, the setup described can easily be modified to perform (simultaneous) measurements at several narrow spectral bands.

Presently, the detector-type required is commercially offered mainly in the visible and near infrared. However, promising research work has also been reported for the mid infrared and the terahertz regimes. Progress in this area combined with the method described here will eventually open new spectral ranges for investigating pulsars.

Acknowledgements. It is a pleasure to acknowledge discussions with Bettina Posselt. We also thank Rudi Dutter for his invaluable help in programming "R".

\section{Appendix A: Signal-to-noise ratio of the histogram peak at $t_{i, j}=T$}

For a numerical characterization of the possibility of detecting a periodic structure in the envelope of the histogram, we concentrate on the histogram bin at $t_{i, j}=T$, showing peaks in frequency $H$, as it is caused by the autocorrelation of the main peak of the optical input signal $s(t)$. In this bin we designate the pulsar contribution $H_{\mathrm{p}}$ and the noise contribution $H_{\mathrm{n}}\left(H=H_{\mathrm{p}}+H_{\mathrm{n}}\right)$. The signal-to-noise ratio in this bin, called $S N_{\text {Hist }}$, may serve as a first measure of how easy it is to find the desired periodicity. We thus have

$S N_{\text {Hist }}=\frac{H_{\mathrm{p}}}{\sigma\left(H_{\mathrm{n}}\right)}$,

where $\sigma\left(H_{\mathrm{n}}\right)=\sqrt{H_{\mathrm{n}}}$ represents the noise, given by the standard deviation of the frequency of the noise events. For a rough 
calculation we now assume the relevant peak of the pulsar signal $s(t)$ to be rectangular with width $d$ and obtain

$S N_{\text {Hist }}=\left(n_{\mathrm{p}} y\right)^{2} \frac{\sqrt{P \cdot b w}}{d} \cdot \frac{1}{\sqrt{n_{\mathrm{n}}\left(T n_{\mathrm{n}}+2 n_{\mathrm{p}}-1 / P\right)}} \cdot$

Here $n_{\mathrm{p}}$ is the number of pulsar events per pulsar period $T$, $y$ is the fraction of $n_{\mathrm{p}}$ contained in that part of $s(t)$ leading to the histogram peak under consideration, $P$ the number of pulsar periods recorded, $b w$ the histogram bin width, and $n_{\mathrm{n}}$ the rate of noise events. Equation (A.2) is obtained under the assumption $n_{\mathrm{p}} y P \gg 1$. For the histograms shown in Figs. 5-7, $S N_{\text {Hist }}$ equals $33,8.1$, and 0.55 , respectively. To calculate these numbers, we used an effective width of the first pulsar peak of $d=3 \mathrm{~ms}$, as estimated from Fig. 4 .

Equation (A.2) indicates that increasing $b w$ is of advantage. However, as soon as $b w$ exceeds the pulse width $d$, the histogram peaks will not longer be resolved. As a result, a practical limit may be given by $b w=d$. The a priori quadratic dependence on the effective number of pulsar events, $n_{\mathrm{p}} \cdot y$, was to be expected for the method involving an autocorrelation. The second appearance of the term $n_{\mathrm{p}}$ in the denominator reflects the generation of histogram noise by the interaction of pulsar events with noise events. Finally, the $\sqrt{P}$-dependence is also to be expected for this type of signal processing. For given period length $T$ it means that $S N_{\text {Hist }}$ increases only proportional to the square root of the measurement time $P T$.

\section{Appendix B: Improvement of signal-to-noise ratio by superimposing histogram blocks of length $T$}

If one further capitalizes on the (sort-of) periodicity within the histogram itself, the signal-to-noise ratio of the envelope can be improved considerably, as demonstrated in Figs. 6 and 7.
To this end we superimpose $k$ consecutive histogram blocks of length slightly larger than $T$. The integer $k$ counts the periods in the histogram, starting at the left, and may be chosen according to $1 \leqslant k \leqslant P$. When asking for the signal-to-noise ratio $S N_{\text {Sup }, k}$ of the peak in the resulting envelope, we have to consider that the pulsar contributions decrease proportional to $(1-k / P)$, while the noise contributions decrease only by $\sqrt{\sum_{k}(1-k / P)}$. Therefore superimposing $k$ blocks improves the signal-to-noise ratio of the first block, $S N_{\text {Hist }}$, to

$S N_{\text {Sup }, k}=S N_{\text {Hist }} \cdot \sqrt{\sum_{k}(1-k / P)}$.

For the typical case $k \gg 1$ and $P \gg 1$, Eq. (B.1) shows that an improvement of $50 \%$ of the maximally achievable value of $\sqrt{P / 2}-$ the latter when choosing $k=P-$ can already be obtained for $k / P=0.134$.

\section{References}

Atwood, W. B., Ziegler, M., Johnson, R. P., \& Baughman, B. M. 2006, ApJ, 652, L49

Cordes, J. M., Kramer, M., Lazio, T. J. W., et al. 2004, New Astron. Rev., 48, 1413

Eikenberry, S. S., Fazio, G. G., Ransom, S. M., et al. 1997, ApJ, 477, 465

Germanà, C., Zampieri, L., Barbieri, C., et al. 2012, A\&A, 548, A47

Larsson, S. 1996, A\&AS, 117, 197

Leeb, W. R., Poppe, A., Hammel, E., et al. 2013, Astrobiology, 13, 521

Mignani, R. P. 2012, in Electromagnetic Radiation from Pulsars and Magnetars, eds. W. Lewandowski, O. Maron, \& J. Kijak, ASP Conf. Ser., 466, 3

Naletto, G., Barbieri, C., Occhipinti, T., et al. 2009, A\&A, 508, 531

Patat, F. 2003, A\&A, 400, 1183

Percival, J. W., Biggs, J. D., Dolan, J. F., et al. 1993, ApJ, 407, 276

Sandberg, A., \& Sollerman, J. 2009, A\&A, 504, 525

Véron-Cetty, M. P., \& Woltjer, L. 1993, A\&A, 270, 370

Zampieri, L., Čadež, A., Barbieri, C., et al. 2014, MNRAS, 439, 2813

Ziegler, M., Baughman, B. M., Johnson, R. P., \& Atwood, W. B. 2008, ApJ, 680, 620 